\title{
MICROWAVE SURFACE RESISTANCE OF ND FILMS
}

\author{
T. Yogi and J. E. Mercereau
}

Low Temperature Physics 63-37

California Institute of Technology, Pasadena, CA 91125

\section{Abstract}

The surface resistance, $R_{s}$, of niobium (Nb) films has been experimentally investigated as a function of thickness, preparation technique and substrate material at $8.86 \mathrm{GHz}$. $\mathrm{Nb}$ films were prepared by either sputtering or evaporation in the thickness range between 0.1 $\mu \mathrm{m}$ and $3.0 \mu \mathrm{m}$ on either copper $(\mathrm{Cu})$ or sapphire substrate. $R_{S}$ was determined using a cylindrical $\mathrm{TE}_{01 \mathrm{I}}$ mode resonant cavity with one removable end-plate which was utilized as the test substrate. The low field $R_{S}$ at $4.2 \mathrm{~K}$ is lower than that of bulk $\mathrm{Nb}$ and shows good agreement with BCS calculation which takes into account the effects of mean free path. The temperature dependence of $\mathrm{R}_{\mathrm{s}}$ indicates a normalized film gap parameter, $\Delta(0) / K_{C}$, nearly equivalent to the bulk value for most of the films. At low temperatures, $R_{s}$ is dominated by residual resistance $\left(R_{0}\right)$ which approaches $1 \mu \Omega$. The overall characteristics of $\mathrm{Nb}$ on $\mathrm{Cu}(\mathrm{Nb} / \mathrm{Cu})$ indicate that this composite material is potentially useful in applications requiring high $\mathrm{rf}$ field as well as high. thermal stability.

\section{Introduction}

At present, only two superconducting materials are utilized in high power microwave applications, $\mathrm{Nb}$ and lead $(\mathrm{Pb})$. If properly processed, these two materials maintain low surface resistance up to high microwave surface fields which approach the thermodynamic critical field, $\mathrm{H}_{\mathrm{c}} \cdot$ Consequently, resonant cavities fabricated from these superconductors have very low loss and are being used, for example, in the development of nuclear particle accelerators. 1,2

$\mathrm{Nb}$ is technically superior to $\mathrm{Pb}$ by approximately a factor of 2 at $10 \mathrm{GHz}$ in terms of the relevant superconducting parameters, $\mathrm{H}_{\mathrm{c}}$ and $\mathrm{R}_{\mathrm{s}}$. It is normally uti-

lized in bulk form or in electron-beam welded structures which require relatively elaborate surface electropolish and high temperature vacuum anneal. ${ }^{3} \mathrm{~Pb}$ has been found to require less elaborate surface processing. In fact the theoretical limiting. $\mathrm{H}_{\mathrm{C}}$ and $\mathrm{R}_{\mathrm{S}}$ can be realized on chemically polished, electrodeposited $\mathrm{Pb}$ layers only a few microns thick. ${ }^{4}$ Thus, superconducting $\mathrm{Pb}$ resonators are commonly copper, $\mathrm{Cu}$, structures ${ }^{5}$ with only a surface layer of $\mathrm{Pb}$, a few microns thick. The basic $\mathrm{Cu}$ structure of these resonators provides relatively great thernal stability. Resonators of this type commonly do not exhibit "run-away" thermal breakdown and consequently multipacting levels are easily processed away.

These preceeding features suggest that thin layers of $\mathrm{Nb}$ (or the $\mathrm{A}-15$ compounds) on $\mathrm{Cu}(\mathrm{Nb} / \mathrm{Cu}$ ) might form a technically superior microwave superconductor. In order to investigate this possibility, research has been initiated to study the surface resistance and critical field of $\mathrm{Nb}$ and $\mathrm{A}-15$ films at microwave frequency. Such a system can be prepared by evaporation, sputtering, or electroplating and has been studied for its dc or very low frequency ac properties. ${ }^{6}, 7$ Only Iimited $\mathrm{r} f$ or microwave study, however, has been performed on this system in the past. ${ }^{8}$ This report is a brief sumary of our preliminary results at $\mathrm{x}$-band for $\mathrm{Nb}$ films.

\section{Experimental Procedure}

Films used in this study were produced either by sputtering or evaporation. Substrates were either OFHC $\mathrm{Cu}$ or sapphire (of optical window quality supplied by Union Carbide) of disc shape; $6.4 \mathrm{~cm}$ in diameter and $3.2 \mathrm{~mm}$ in thickness. After machining, Cu substrates were lapped on emery paper of successively finer grit; the final mechanical polish was accomplished with levigated alumina compound of 1-3 $\mu \mathrm{m}$ in particle size. Prior to deposition by either method, $\mathrm{Cu}$ discs were electropolished to remove several $\mu \mathrm{m}$ of surface material. Sapphire substrates were cleaned by conventional chemical cleaning agent. Sputtered samples

$\mathrm{Nb}$ films were sputtered onto $\mathrm{Cu}$ substrates to the thickness of $0.33,1.0$ and $3.0 \mu \mathrm{m}$. Sputtering was performed in a planar diode configuration with targetsample distance of $4.5 \mathrm{~cm}$ using an rf power source. First, the substrate was sputter-etched for $\sim 1500 \AA$ to remove the surface oxide. Then $\mathrm{Nb}$ was sputtered onto the substrate at a typical rate of $300 \AA / m i n$ in a 10 micron argon atmosphere. During etching and deposition the substrate temperature was estimated to have risen to about $350^{\circ} \mathrm{C}$. Evaporated samples

$\mathrm{Nb}$ films were evaporated on $\mathrm{Cu}$ substrates at $0.11,0.33$ and $0.80 \mu \mathrm{m}$ in thickness and on sapphire at $0.33 \mu \mathrm{m}$. Substrates were heated to $\simeq 400^{\circ} \mathrm{C}$ in the vacuum chamber (Ultek, all metal, ion pumped) prior to eyaporation and maintained near that temperature throughout evaporation. Base pressure was typically. below $10^{-8} \mathrm{~mm} \mathrm{Hg}$ and chamber pressure remained below $2 \times 10^{-7} \mathrm{~mm} \mathrm{Hg}$ during electron-beam evaporation. Typical average evaporation rate was $300 \AA / m i n$. Microwave measurement

The $\mathrm{x}$-band cylindrical resonator used for this study was made of OFHC copper with a diameter/length ratio of 1.75 and was operated at $8.86 \mathrm{GHz}$ in $\mathrm{TE}_{01 \mathrm{l}}$ mode. ${ }^{15}$ All measurements were performed in a pulsed mode in which resonator $Q$, field level and energy content could be determined by measuring the decay time togethex with the incident, reflected and transmitted powers. For a homogeneous resonator $R_{S}=T / Q$ where the geometrical factor $\Gamma$ is $708 \Omega$ for the present geometry. For these measurements the entire resonator was first electroplated with $\mathrm{Pb}$ to a thickness of $3 \mu \mathrm{m}$ and relevant parameters such as $Q$ and its field dependence were determined to establish a "baseline" for $\mathrm{Pb}$. Then the bottom plate of the resonator was replaced with a thin film sample plate for a similar set of measurements. The surface resistance of the thin film sample bottom plate, $R_{1}$, is related to the measured quality factors by:

$$
R_{1}=\frac{\Gamma}{Q_{0}}\left[1+\frac{1}{f}\left(\frac{Q_{0}}{Q_{1}}-1\right)\right]
$$

where $Q_{0}$ is the baseline quality factor of entirely $\mathrm{Pb} / \mathrm{Cu}$ surface and $\mathrm{Q}_{1}$ is that of the same cavity with the thin film sample bottom plate. The parameter $f$ is the fraction of the loss arising from the bottom plate 
and is 0.237 for our present geometry. Transition temperature and resistivity In order to assess the quality of the sputtered and evaporated films, the transition temperature $T_{c}$ was determined inductively at low frequency $(\sim 400 \mathrm{~Hz})$ by measuring the coupling between a pair of coils placed within $\sim 1 \mathrm{~mm}$ of the sample surface. The sample was thermally anchored to a copper block which was placed in a partially evacuated chamber immersed in a helium bath. The temperature of the copper block and the sample was controlled by a heater and was monitored by a germanium thermometer. By this method the $\mathrm{T}_{c}$ of every sample was determined. For evaporated films, small samples were made on sapphire substrates at the same time as the disc samples were produced. The resistivity, $\rho$, as well as $\mathrm{T}_{c}$ of these small samples were subsequently determined by a 4-point probe measurement in order to deduce the mean free path of the electrons, \&, using a standard relationship between $\rho$ and 2.13

\section{Results and Discussion}

This report presents data on several niobium films relating microwave surface resistance $R_{S}$ at 8.86

$\mathrm{GHz}$ to various film parameters.

Transition temperature

The films (see Table 1 ) range in thickness $\mathrm{d} \simeq$ $.1-3 \mu \mathrm{m}$. In this range of thickness the proximity effect of the substrate is small, except for the thinnest films. Target material for the films was $0.999 \mathrm{Nb}$ with $\mathrm{T}_{\mathrm{c}}=9.25 \mathrm{~K}$. Precision of the temperature measurement is estimated to be $\pm 0.025 \mathrm{~K}$ based on calibration from $T_{c}$ of high purity $N b$ and $\mathrm{Pb}$. We found $T_{c}$ for sputtered films. on $\mathrm{Cu}$ to be genera11y somewhat higher than that of the target material, and that it increased with increasing thickness. Typical transition width $(10 \%-90 \%)$ was $\Delta \mathrm{T} \simeq \pm 0.020 \mathrm{~K}$. By comparison, evaporated $\mathrm{Nb}$ films on $\mathrm{Cu}$ had slightly lower transition temperatures (by $\sim 0.1 \mathrm{~K}$ ) than sputtered films of equivalent thickness. Transition temperature data is listed in Table 1 for several films. Note that for thin evaporated films of equal thickness, $T_{c}$ on sapphire is

slightly higher than on $\mathrm{Cu}$-- probably reflecting the proximity effect in the thinner films.

The highest $\mathrm{T}_{\mathrm{c}}$ observed was for $3 \mu \mathrm{Nb}$ sputtered on $\mathrm{Cu}$ where $\mathrm{T}_{\mathrm{c}}=9.74 \mathrm{~K}$ or $0.5 \mathrm{~K}$ above that of the target material. The highest temperature for the onset of superconductivity in this film was $9.89 \mathrm{~K}$. This modest increase in $\mathrm{T}_{c}$ is outside the range of experimental uncertainty and we believe it to be real. Low field surface resistance

For all films and substrates $R_{S}(T)$ was found to

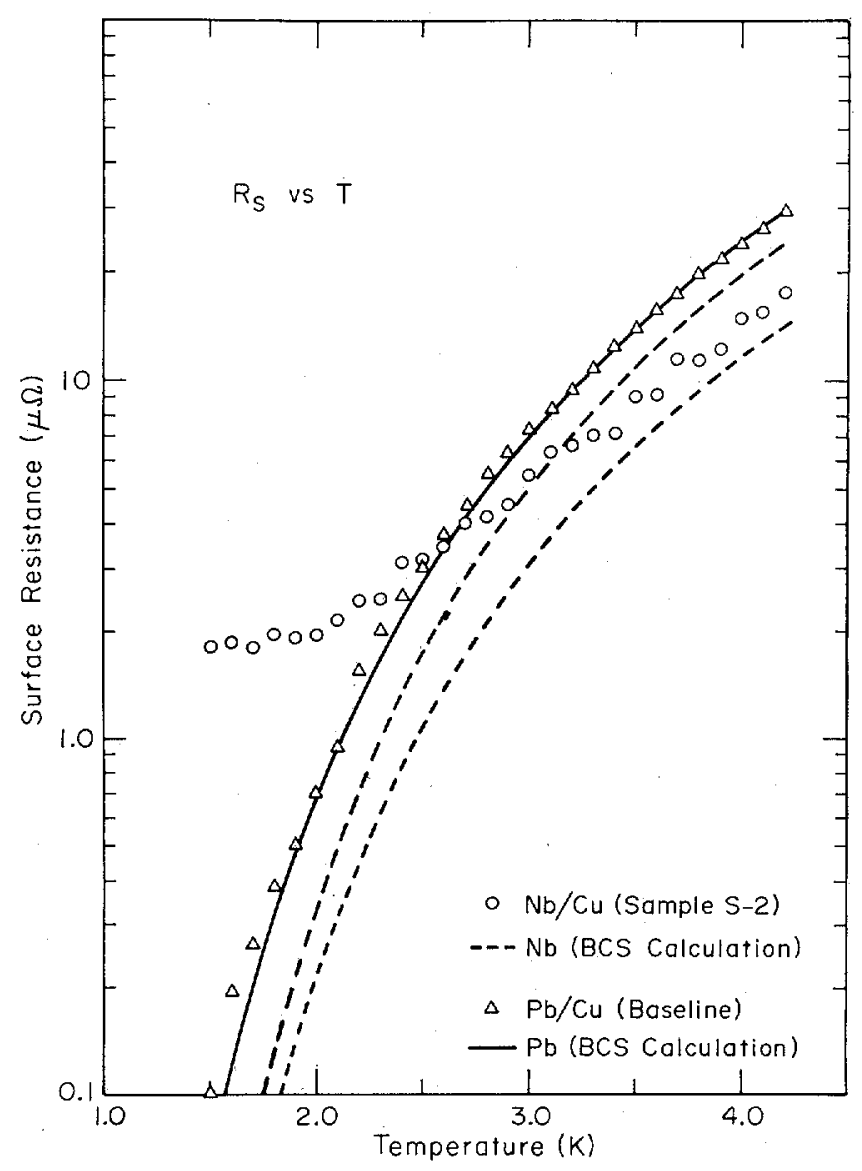

Fig. 1 Surface resistance as a function of temperature. BCS surface resistance is plotted for both $\mathrm{Pb}$ and $\mathrm{Nb}$ for comparison. Dashed curves (upper: $\ell=$ 10,000 $\AA$, specular. Lower: $\ell=300 \AA$, diffuse).

depend somewhat on peak microwave surface field $h_{s}$. However, at low fields $R_{s}(T)$ is independent of $h_{s}$. At higher microwave fields $\mathrm{R}_{\mathrm{S}}$ and the microwave critical field show a very strong dependence on substrate material as will be published elsewhere.

The temperature dependence of $R_{s}$ presented here is for peak surface fields $\lesssim 0.3 \mathrm{mT}$. which is well within the linear range of $\mathrm{R}_{\mathrm{s}}$ for all films.

Figure 1 indicates (as triangles) data on $R_{S}(T)$ for $\mathrm{Pb} / \mathrm{Cu}$ as weli as typical data for $\mathrm{Nb} / \mathrm{Cu}$ films (as circles). The $\mathrm{Pb} / \mathrm{Cu}$ data comes from measurements on a resonator fabricated entirely of $\mathrm{Pb} / \mathrm{Cu}$ as discussed previously. The $\mathrm{Pb} / \mathrm{Cu}$ data is compared with a BCS

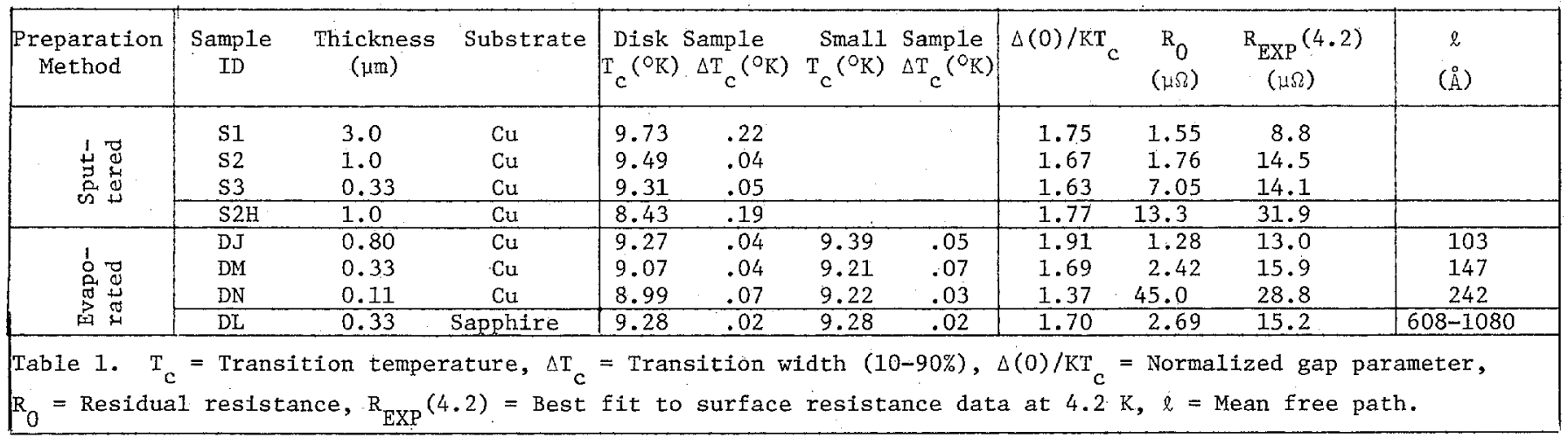


calculation ${ }^{9}$ as the solid curve and shows very good agreement over this entire temperature range 0.2 < $\mathrm{T} / \mathrm{T}<0.6$ ) with little residual resistance (excess resistance above $\mathrm{BCS}$ ) at the lowest temperature. At the lowest temperature $(\simeq 1.5 \mathrm{~K}) \mathrm{Pb} / \mathrm{Cu}$ surfaces typically showed a residual resistance less than $0.1 \mu \delta$. The $\mathrm{Nb}$ data is from measurements on the same resonator with a $\mathrm{Nb}$ film endplate, as analyzed in Section II. The dashed curves are the BCS calculated values for $R_{s}$ utilizing the accepted materials parameters for $\mathrm{Nb}, 11$ The difference between the two curves is due to mean free path effects (discussed 1ater) and represent the extreme limits on $R_{S}$ due to this effect. In general these $\mathrm{Nb}$ films show the ideal BCS surface resistance at the highest temperature $\left(T \sim 4.2 \mathrm{~K}, \mathrm{~T} / \mathrm{T}_{\mathrm{c}} \sim 0.45\right)$ but a relatively large residual resistance at lower temperature.

Further comparison between $\mathrm{Nb}$ film data and the BCS calculation was made by performing a least-squares fit to the following equation: ${ }^{10}$

$$
R_{S}(T)=A \text { exp }\left(-\frac{\Delta(0)}{K T_{c}} \frac{T c}{T}\right)+R_{0}
$$

where the first term represents an approximation to the BCS surface resistance below $\mathrm{T} \leq 0.5 \mathrm{~T}_{\mathrm{c}}$ and the second term accounts for a temperature insensitive residual resistance, $R_{0}$. The parameters utilized in fitting this equation to the data were $A$, zero temperature gap $\Delta(0)$ and residual resistance $R_{0}$. Figure 2

illustrates surface resistance data fit in this way but $R_{s}$ is now plotted against $T_{c} / T$. Raw data are

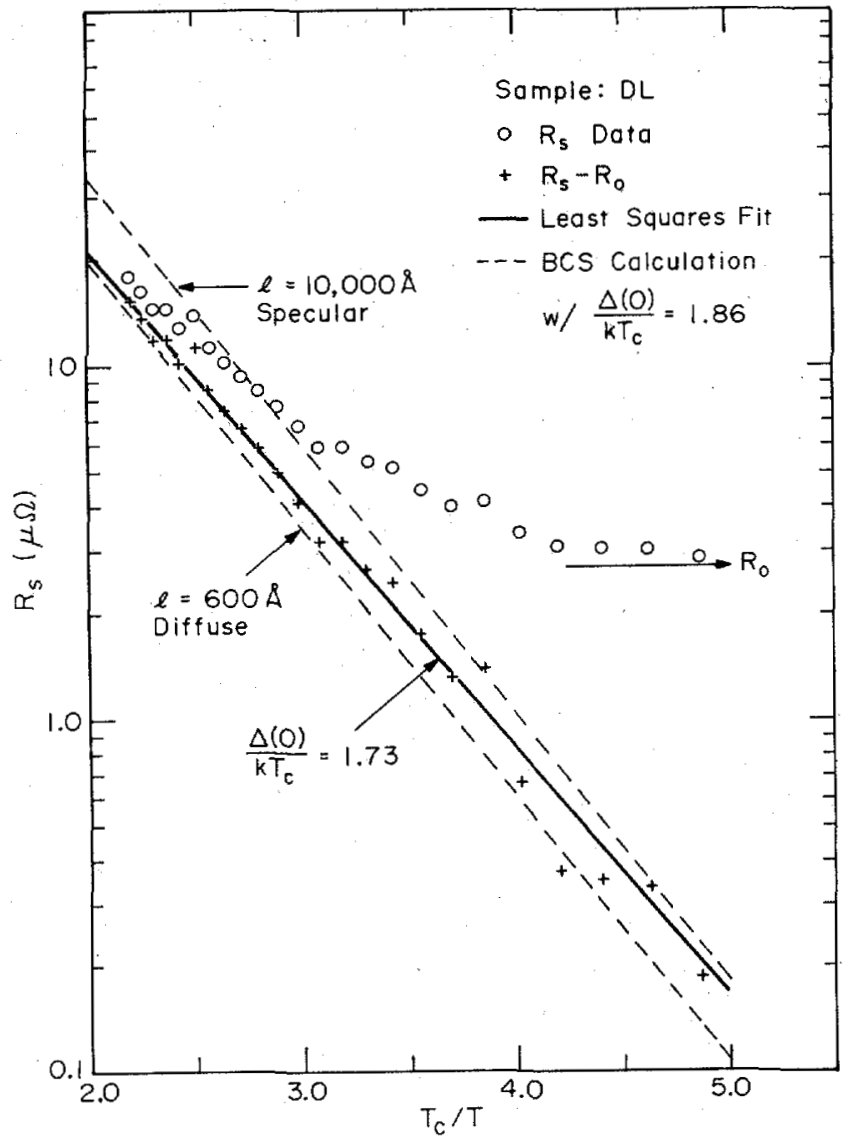

Fig. 2 Surface resistance for $\mathrm{Nb}$ films plotted against the reciprocal of normalized temperature. Dashed lines are BCS calculations (see text). plotted on circles while the crosses represent the raw data minus a residual resistance factor. The solid line is a least square fit evaluating $\Delta(0)$. In Table 1 these parameters are listed for several films. Also listed as $R_{E X P}(4.2)$ is the best ift for $R_{s}-R_{0}$

from Equation (2). These quantities are discussed below.

Gap parameter. For bulk Nb cavities, previous microwave measurements of $\mathrm{R}_{\mathrm{S}}(\mathrm{T})$ indicate a normalized gap parameter, $\Delta(0) / \mathrm{KT}_{\mathrm{c}} \simeq 1.85 ;^{10}$ previous tunneling data on $\mathrm{Nb}$ films gives $\Delta(0) / \mathrm{KT} \mathrm{c}_{\mathrm{c}} \sim 1.9 .12$ As shown in Table 1, our data, utilizing $R_{s}$ from these $\mathrm{Mb} / \mathrm{Cu}$ films, leads to a normalized gap parameter $\sim 1.8$ which is a slight function of thickness and is generally larger for the films with lower $R_{0}$.

Ideal surface resistance. The BCS surface resistance can be extracted from Equation (2) as $R_{S}(T)-R_{0}$. This quantity has been calculated ${ }^{10}$ and is a function of mean free path $l$. The theory predicts a minimum in $R_{S}(T)-R_{0}$ when $\ell$ is about equal to the London penetration depth, $\lambda_{L}$, which is $\approx 330 \AA$ for $\mathrm{Nb}$. This minimum becomes more pronounced at low frequency. The calculated 9 dependence of $R_{s}(T)-R_{0}$ on $\&$ for $\mathrm{Nb}$ at $4.2 \mathrm{~K}$ and at $8.86 \mathrm{GHz}$ is shown as curves in Figure 3 , using standard material parameters. ${ }^{11}$ Also indicated are experimental evaluations of this quantity, $R_{\mathrm{Exp}}$

(see Table 1), shown as a function of mean free path for several evaporated films. For these films the mean free path was deduced from the measured resistivity, just above $T_{c}$, of small samples on sapphire substrates produced during the same evaporation. Except for the thinnest $(.11 \mu \mathrm{m})$ film the experimental values for $R_{\operatorname{Exp}}$ are consistent with the theoretical calculation. Note that this resistance is less than for bulk $\mathrm{Nb}$ which typically has a larger \& than the present films. For the thinnest film we suspect only partial screening of the surface current by the film as will be discussed later.

For sputtered films, experimental values for mean free path were not available; consequently $\mathrm{R}_{\mathrm{EXP}}$ data are indicated only at the right side of Figure 4 as

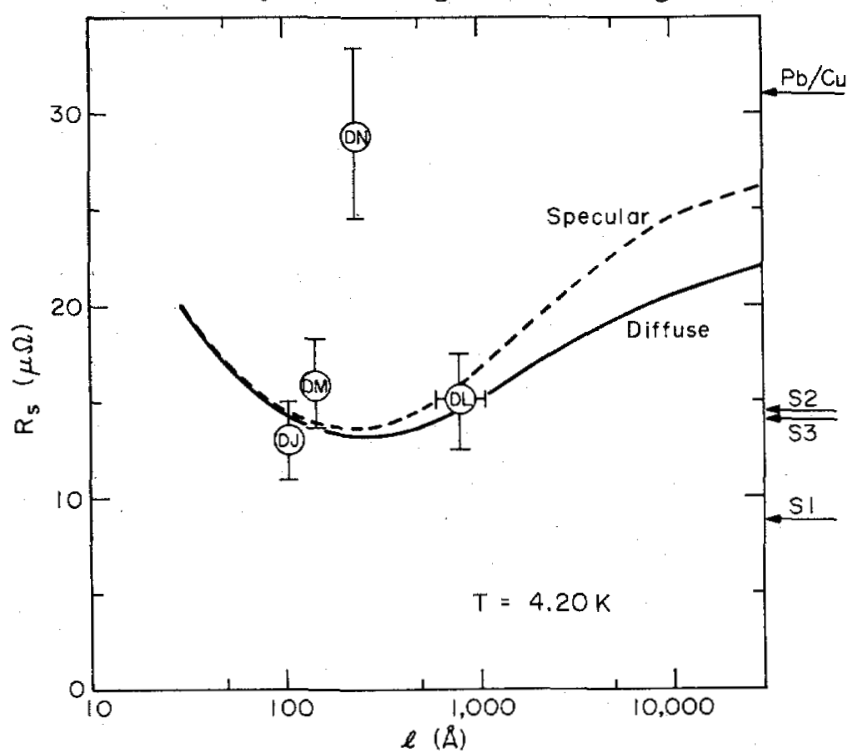

Fig. 3 Surface resistance of $\mathrm{Nb}$ films at $4.2 \mathrm{~K}$ as a function of electronic mean free path and surface reflection. 
S1, S2, and S3 (see Table 1). These data imply a short mean free path: S2 and S3 indicate that $100 \leq$ $\ell \leq 1000 \AA$. The thick sputtered film, $S 1$, was found to have anomalously small $R_{\text {EXP }}$ which is $\simeq 30 \%$ below the minimum value given by the calculation; this film also has the highest transition temperature (see Table 1 ).

Residual resistance. For these films $\mathrm{R}_{0}$ is typ-

ically a few micro-ohms. The residual resistance increases as thickness decreases and for equivalent thickness is lower for the evaporated films. The thinnest evaporated film $(d=.11 \mu)$ is only approximately two penetration depths thick and the relatively large $R_{0}$ is due to flux leakage into the substrate.

A photomicrograph of a typical $0.8 \mu \mathrm{Nb}$ film (DJ in Table 1) on $\mathrm{Cu}$ substrate is shown in Figure $4(\mathrm{a})$ where the line structures are the grain boundaries of the Cu substrate. Stall pits, also shown in Figure 4 (a) are typically a few $\mu \mathrm{m}$ in diameter, and are predominantly located along grain boundaries. In order to determine the uniformity of $\mathrm{Nb}$ coverage, random areas of several samples were scanned under SEM searching for $\mathrm{x}$-ray emission from $\mathrm{Cu}$. The result showed no discontinuity in the $\mathrm{Nb}$ coverage across grain boundaries and in general $\mathrm{Nb}$ coverage was complete even for the thinnest sputtered and evaporated films.

On the other hand some of the pits such as the one shown in the middle of Figure 4 (b) showed an excessive amount of $\mathrm{Cu}$ indicating that these pits were not entirely covered with $\mathrm{Nb}$. This type of imperfection can easily give rise to $R_{0}$ via normal metal

dissipation. We estimate that such norma1 spots, if present at a fractional level of $\sim 2 \times 10^{-4}$, would lead to $R_{0} \sim 1 \mu \Omega$ (the normal state surface resistance of

$\mathrm{Cu}$ was measured to be $5.6 \mathrm{~m} \Omega$ ). Thus at this grain density, only one pit in ten grains could account for the observed $\mathrm{R}_{0}$. Generally $\mathrm{R}_{0}$ decreases as the thickness is increased for both preparation methods, which seems to indicate that the $\mathrm{Nb}$ coverage improves with thickness.

An attempt was made to anneal one of the samples (S2) above the stress annealing temperature $\left(750^{\circ} \mathrm{C}\right.$ ) of Nb. It was annealed at $800^{\circ} \mathrm{C}$ for $\sim 3$ hours in a vacuum oven at $<5 \times 10^{-6} \mathrm{~mm} \mathrm{Hg}$. The heat treatment resulted in a marked decrease in $\mathrm{T}_{\mathrm{c}}$ of the film from 9.49 to $8.43 \mathrm{~K}$ and at the same time considerable increase in $R_{0}$ as well as $R_{\text {EXP }}(4.2)$, as entered in Table 1 by $\mathrm{S} 2 \mathrm{H}$. One possible cause of this behavior is the partial diffusion of $\mathrm{Cu}$ into $\mathrm{Nb}$ which reduces the mean free path to increase the BCS part of surface resistance (to the left side of Figure 4) and at the same time increases the effective penetration depth so that the surface current leaks into the substrate resulting in an increased $R_{0}$.

When the film thickness becomes comparable to effective penetration depth $(400-800 \AA$ for these $\mathrm{Nb}$ films), because of field leakage into the substrate

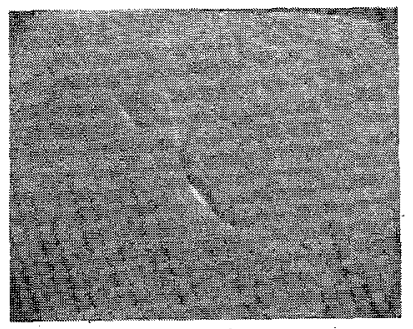

(a) $\quad 20 \mu \mathrm{m}$

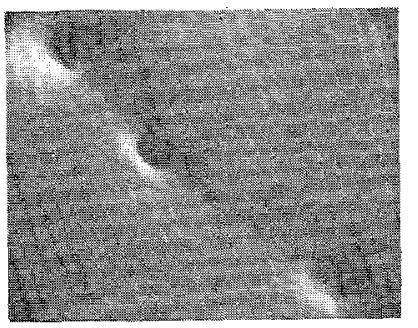

(b) $\stackrel{5-r}{2 \mu m}$
$\mathrm{Cu}$, the surface resistance becomes a function of thickness. It is possible to estimate the approximate magnitude of this effect by calculating the effective penetration depth of $\mathrm{Nb}$ for a known mean free path using the same computer program described earliex. The results show that except for the very thinnest film used (sample DN), the current leakage should give no more than $0.2 \mu \Omega$ in $R_{0}$. However for the thinnest

film sample DN, these calculations indicate that a major fraction of $R_{0}(45 \mu \Omega)$ is due to this effect.

Finally it should also be pointed out that there are some variables that depend on the processing method since two films of the same thickness produced by sputtering versus evaporation give significantly different $R_{0}$ (7.05 $\mu \Omega$ for $S 3$ as opposed to $2.42 \mu \Omega$ for $D M)$.

A-15 Compound

A natural extension of this technique is to superconducting films of A-15 compounds on $\mathrm{Cu}$ substrates. Preliminary investigation has been initiated in this direction by forming $\mathrm{Nb}_{3} \mathrm{Au}$ compound in two ways. One method is by evaporating a layer of Au (typically $2700 \AA$ ) between two $\mathrm{Nb}$ layers (typically $3500 \AA$ ) on $\mathrm{Cu}$ substrate and subsequent1y heat treating at $\sim 700^{\circ} \mathrm{C}$ in vacuum for several hours. Another method is by sputtering a $1 \mu \mathrm{m}$ layer of Au on bulk $\mathrm{Nb}$ followed by a similar heat treatment. Films thus produced show transition temperatures of $10.79 \mathrm{~K}$ and $10.89 \mathrm{~K}$, respectively, which are close to the published $\mathrm{T}_{c}(=11.0 \mathrm{~K})$ of bulk $\mathrm{Nb}_{3} \mathrm{Au}^{1{ }^{14}}$ Microwave measurement and surface analysis of films thus produced are underway.

\section{References}

1. J. R. Delayen et al., this conference.

2. K. W. Shepard et a1., IEEE Trans. MAG-15, 666 (1979).

3. H. Pfister, Cryogenics 16, 17 (1976).

4. T. Yogi, Ph.D. Thesis (California Institute of Technology, 1976).

5. G. J. Dick and G. D. Sprouse, IEEE Trans. MAG-13, 512 (1977).

6. R. W. Meyerhoff and W. T. Beall Jr., J. AppI. Phys. 42, 147 (1971).

7. M. J. Witcomb, Phys. Stat. Sol.(a) 42, 595 (1977)

8. H. Padamsee et al., IEEE Trans. MAG-13, 346 (1977). Also private communication.

9. J. Halbritter, Kernforschungszentrum Karlsruhe, Externer Bericht 3/70-6 (June 1970).

10. J. Halbritter, Z. Physik 238, 466 (1970).

11. P. Wilson, SLAC-TN-70-35.

12. M. R. Beasley, in Euture Trends in Superconductive Electronics, ed. by B. S. Deaver et a1., American Institute of Physics (1978), p. 389396.

13. A. F. Mayadas et a1., J. App1. Phys., 43, 1287 (1972).

14. E. Bucher et a1., Phys. Lett. 8, 27 (1964).

15. T. Yogi, Internal Report, LTP-AUG-79A, California Institute of Technology.

Fig. 4 Photomicrograph of (a) sample DJ, (b) pits along grain boundaries. 